\title{
Determinantes del mantenimiento del consumo de tabaco: extensión de la Teoría de la Conducta Planificada
}

\author{
Determinants of maintenance snuff consumption: extension of the Theory of Planned \\ Behavior
}

\author{
Mar Durán \\ Mauro Rodríguez \\ María-José Ferraces \\ Adhara Río \\ José-Manuel Sabucedo \\ Universidad de Santiago de Compostela, España
}

Rec (02 septiembre 2015) Acept (25 enero 2016)

\begin{abstract}
Resumen
El objetivo fundamental de este trabajo es explorar la importancia de las emociones positivas y negativas, la norma moral y la conducta pasada en la intención de mantener el consumo del tabaco más allá de la explicación dada por la Teoría de la Conducta Planificada. Se utilizó una muestra final de 202 estudiantes fumadores de la Universidad de Santiago de Compostela, con edades comprendidas entre 18 y 25 años. Los resultados muestran que las variables añadidas aumentan de forma significativa la capacidad explicativa del modelo $\left(\Delta R^{2}=20 \%\right)$. Esto, junto con la relación que presentan estas variables con la intención conductual [emociones positivas $(\beta=.23)$; norma moral $(\beta=-.14)$; conducta pasada $(\beta=.72)$ ], es lo que hace que estas variables adquieran relevancia explicativa en el modelo y nos dote de herramientas necesarias para intervenir y ayudar a mejorar la salud de nustros jóvenes.

Palabras clave: Emociones, norma moral, conducta pasada, intención, uso de tabaco
\end{abstract}

\begin{abstract}
The main objective of this paper is to explore the importance of positive and negative emotions, the moral norm and the past behavior in the intention of keeping the consumption of snuff beyond the explanation given by the Theory of Planned Behavior. A final sample of 202 smokers students at the University of Santiago de Compostela, aged between 18 and 25 years was used. The results show that the added variables significantly increase the explanatory power of the model $\left(\Delta R^{2}=20 \%\right)$. This, together with the list presented by these variables with behavioral intention [positive emotions $(\beta=.23)$; moral norm $(\beta=-.14)$; past behavior $(\beta=$ $.72)$ ], it is what makes these explanatory variables acquire relevance in the model and we dowry of tools needed to intervene and help improve the health of our young's.

Key words: Emotions, moral norm, past behavior, intention, tobacco use.
\end{abstract}

\footnotetext{
Correspondencia: mar.duran@usc.es; mauro.rodriguez@usc.es; mjose.ferraces@usc.es; adhara.rio@gmail.com; josemanuelsabucedo@usc.es Agradecimientos: Este trabajo ha sido realizado con la ayuda GPC2013-017 de la Xunta de Galicia para Grupos de Referencia Competitiva y cofinanciado con fondos FEDER.
} 


\section{Introducción}

A pesar de que la información aportada desde el Ministerio de Sanidad y Consumo en España apunta a una progresiva disminución del número de fumadores, existen estudios que evidencian que, a pesar de este descenso, los datos siguen siendo preocupantes (Catalina, Gelpi, Cortés, Martín, 2010). Un ejemplo de esto también nos lo proporciona la última Encuesta Estatal sobre Uso de Drogas en Estudiantes de Enseñanza Secundaria (Ministerio de Sanidad, Servicios Sociales e Igualdad de España, 2011/2012), donde se pone de manifiesto que el tabaco es una de las substancias más consumidas por los estudiantes españoles. En 2013, el 43.8\% fumó tabaco alguna vez en la vida, el $35.3 \%$ en el último año y el $12.5 \%$ a diario. Otro dato aportado y no menos preocupante, lo encontramos en el hecho de que este tipo de consumo se produce a edades cada vez más tempranas, situándose la edad de inicio en los adolescentes españoles en torno a los 12 años, con un consumo medio diario de 7.4 cigarrillos.

La Asociación Española Contra el Cáncer (2014), revela que el consumo de tabaco es responsable de aproximadamente el $30 \%$ de las muertes por cáncer, del $20 \%$ de las producidas por enfermedades cardiovasculares y del $80 \%$ de las enfermedades pulmonares obstructivas crónicas (EPOC) y esto se hace extensible a la mayoría de los países. Teniendo en cuenta que el consumo de tabaco constituye actualmente la primera causa evitable de mortalidad en todos los países desarrollados (Becoña, 2004), es necesario conocer mejor los factores psicosociales que influyen en el mantenimiento de este comportamiento de riesgo, de forma que se pueda llegar a proponer medidas concretas para intentar evitarlo.

En la investigación llevada a cabo en relación con el consumo de tabaco se han aplicado distintos modelos teóricos, desde el Modelo de Creencias de Salud (Becker, 1974; Rosenstock, 1974), hasta la Teoría de la Acción Razonada (Fishbein y Ajzen, 1975) y la Teoría de la Conducta Planificada (Ajzen y Madden, 1986). Esta última es una de las que más se ha utilizado para intentar explicar los factores que inciden tanto en la intención como en la conducta de fumar (McMillan y Conner, 2003; Spijkerman, van den Eijnden, Vitale y Engels, 2004; NatanGolubev, Shamrai, 2010; Murnaghan, Blanchard, Rodgers, LaRosa, MacQuarrie, MacLellan, Gray, 2010).

De acuerdo con la Teoría de la Conducta Planificada (TCP), el determinante inmediato de la conducta es la intención conductual. Esta, a su vez, está determinada por la actitud hacia la conducta, la norma subjetiva y el control conductual percibido. Estas tres variables se basan, respectivamente, en las creencias conductuales, normativas y de control. Sin embargo, como exponen los autores, existen factores que pueden limitar la voluntad de los sujetos de querer realizar una conducta determinada. Por ello, el control percibido será considerado también, junto a la intención, como un determinante inmediato de la conducta.

A pesar de que la TCP es una de las más utilizadas para predecir una gran variedad de conductas, según un reciente meta-análisis de McEachan, Conner, Taylor y Lawton (2011), su capacidad explicativa en comportamientos de riesgo, como el que aquí se plantea, no ha sido muy elevada (entre el 13.8\% y el 15.3\%), encontrándose relaciones entre las distintas variables del modelo moderadas o poco significativas. Lo mismo sucede con el trabajo de Murnaghan, Blanchard, Rodgers, LaRosa, MacQuarrie, MacLellan y Gray (2010), de ahí que, desde diferentes trabajos, se haya propuesto una extensión del modelo, añadiendo variables adicionales que permita incrementar su poder explicativo.

En el contexto de las conductas de riesgo en general, y en la de fumar en particular, ha habido diferentes propuestas que han considerado, por ejemplo, la autoeficacia (Bermúdez y Contreras, 2008), las normas sociales (Dohnke, WeissGerlach y Spies, 2011; Lazuras, Chatzipolychroni, Rodafinos y Eiser, 2012) o la autoidentidad (Hassandra, Vlachopoulos, Kosmidou, Hatzigeorgiadis, Goudas y Theodorakis, 2011), entre otras. Sin embargo, recientemente se ha abierto una línea en que las emociones ocupan un papel central en el estudio de intenciones de acción y comportamientos convirtiéndose en una variable importante a tener en cuenta en relación con la acción y la intención de fumar (Conner, Sandberg, McMillan y Higgins, 2006; Moan y Rise, 2005; Lazuras, Chatzipolychroni, Rodafinos y Eiser, 2012). Por ello, en este estudio se ha optado por incluir emociones anticipadas debido a que las conductas de riesgo son conductas de alta implicación emocional, que difícilmente pueden ser abordadas en su totalidad desde un modelo de persona básicamente racional. Se han incluido tanto emociones positivas como negativas, la razón fundamental es que en la conducta de fumar se da una cierta complejidad, ya que este tipo de comportamiento presenta tanto aspectos positivos como negativos para la percepción del consumidor. Los primeros pueden resultar más inmediatos y van ligados al placer que toda substancia adictiva causa y los negativos (desarrollo de un cáncer u otro tipo de enfermedades para la salud), ocurrirían a medio o a largo plazo y no se darían siempre.

Otra variable que se ha añadido ha sido la norma moral. Las investigaciones llevadas a cabo para examinar el modelo 
de la TCP incluyendo esta variable como predictor adicional, han aportado un incremento significativo de aproximadamente un $3 \%$ en lo que respecta a la capacidad del modelo para predecir la intención (Moan y Rise, 2005; Rivis, Sheeran y Armitage, 2009; Jellema, Abraham, Schaalma, Gebhardt y Empelen, 2013), de ahí que también se haya incluido en esta investigación.

La última variable que se ha añadido en este estudio al modelo original de Ajzen ha sido la conducta pasada por ser una de las que inciden de manera significativa tanto en la intención como en la acción de fumar (Higgins y Conner, 2010; Moan y Rise, 2005; Conner y Armitage, 1998; Rhodes y Courneya, 2003).

Teniendo en cuenta que estas tres variables, que a priori ayudan en la explicación de este comportamiento, nunca han sido estudiadas de forma conjunta en esta conducta de riesgo, los objetivos principales de este estudio se pueden enmarcar en los siguientes: 1) conocer la posible contribución que las variables añadidas al modelo original aportan en la explicación de la intención de fumar y, 2) especificar el tipo de relación que se establece entre estas nuevas variables y las variables de la TCP.

\section{Método}

\section{Participantes}

Se partió de una muestra inicial de $\mathrm{N}=486$ estudiantes de la Universidad de Santiago de Compostela, quedando la muestra reducida a $\mathrm{N}=202$ fumadores, con edades comprendidas entre los 18 y 25 años, de la cual 35 son hombres (17.2\%) y 167 mujeres (82.8 \%). La media de edad es de 20.53 y la desviación típica de 1.93 .

\section{Instrumento}

Para llevar a cabo esta investigación, se construyó un cuestionario basándonos en las instrucciones dadas por Ajzen $(2002,2006)$. Para la medida de las variables se han utilizado escalas de tipo Likert de 7 puntos, excepto para las actitudes que se utilizó un diferencial semántico. En cada caso concreto, se especificarán los extremos correspondientes.

Para medir la Actitud hacia la Conducta se utilizó una escala de diferencial semántico de 7 puntos compuesta por un único ítem y 5 pares de adjetivos. Se ha tenido en cuenta para ello los dos componentes que presenta la evaluación global del individuo y a los que Ajzen denomina instrumental (perjudicial-beneficioso) y otro más experimental (agradabledesagradable) respectivamente. "Para usted, fumar es...", "beneficioso/perjudicial, agradable/desagradable, útil/ inútil, bueno/malo, divertido/aburrido".

Para medir la Norma Subjetiva (NS), se formularon 3 ítems intentando recoger las expectativas que el sujeto cree que tienen sus grupos significativos: "La mayoría de la gente que me importa piensa de mí que (yo deberíalyo no debería) fumar durante los próximo 60 días". "La gente que me importa espera de mí que fume durante los próximos dos meses (extremadamente probable/extremadamente improbable"). "La gente cuya opinión valoro (aprobaria/desaprobaría) que yo fumase durante los próximos dos meses".

Según Ajzen (2002), la escala de Control Conductual Percibido (CCP) deberá contener ítems de auto-eficacia y controlabilidad. En base a esto, se utilizaron 4 ítems, 2 de autoeficacia: "Para usted, fumar durante los próximos 60 días es...". "Si usted quisiera, podría fumar durante los próximos 60 días", entre "totalmente posible" y "totalmente imposible" y entre "completamente cierto" y "completamente falso", respectivamente. Y otros dos de controlabilidad: “¿Cuánto control cree que tiene sobre el hecho de fumar durante los próximos 60 días?". "Depende casi totalmente de usted el hecho de que usted fume durante los próximos 60 días", entre "no tengo control" y "tengo completo control" y entre "totalmente en desacuerdo" y "totalmente de acuerdo", respectivamente.

Para conocer la intención, se utilizaron 2 ítems: "Tengo intención de fumar durante los próximos 60 días". "Intentaré fumar durante los próximos 60 días", entre "extremadamente improbable" y "extremadamente probable" en el primero de ellos y entre "definitivamente falso" y "definitivamente verdadero", en el segundo.

Como ya se ha señalado anteriormente, se han añadido tres nuevas variables a las clásicas de la TCP. Así, para medir las emociones, se ha utilizado un único ítem que recoge 9 emociones, tanto positivas como negativas: "Si en los próximos 60 días fumases, sentirías: enojo, culpabilidad, entusiasmo, miedo, alegría, vergüenza, arrepentimiento, confianza y tristeza". En este caso la escala va desde $1=$ nada a $7=$ del todo.

Para la medición de la norma moral (NM), se utilizaron 3 ítems entre "totalmente en desacuerdo" y "totalmente de acuerdo": "Me siento moralmente obligado a no fumar". "Fumar va contra mis principios morales". "Independientemente de lo que hagan los demás, mis valores me dicen que no debo fumar". 
La última variable, la conducta pasada $(\mathrm{CP})$, fue medida con un solo ítem y se formuló del siguiente modo: “ ¿Con qué frecuencia has fumado durante los últimos 60 días?", entre "nunca" y "siempre".

Procedimiento El cuestionario se pasó de manera colectiva durante el horario lectivo. En todo momento se garantizó el anonimato y la confidencialidad de las respuestas de los participantes, haciendo especial hincapié en la importancia de la sinceridad de las respuestas.

Análisis de datos Para establecer la posible dimensionalidad de las emociones anticipadas, se realizó un análisis factorial exploratorio y se obtuvieron dos factores significativos. Por un lado, un factor de emociones positivas (EPA), compuesto por entusiasmo y alegría, y otro, de emociones negativas (ENA) formado por culpabilidad, arrepentimiento, tristeza y vergüenza. Ambos explicaron el $77.42 \%$ de la varianza.

Después, se realizó un análisis de los coeficientes $\alpha$ de fiabilidad y las correlaciones entre las variables (véase Tabla 1). Posteriormente, se procedió a la realización de dos regresiones jerárquicas en las que la variable dependiente era la intención de fumar. En el Paso 1 se analizaron las variables de la TCP. Posteriormente, se incluyeron las emociones positivas y negativas (EPA y ENA), la norma moral (NM) y la conducta pasada (CP), para conocer la contribución que todas ellas hacían a la intención de fumar (véase Tabla 2).

Se comprobaron los estadísticos de la independencia de los residuos mediante la prueba de Durbin-Watson, donde se obtuvieron valores de 2,$56 ; 2,47 ; 2,57 ; 2,33$ y 2,45 , respectivamente (no existe auto-correlación). Al analizar conjuntamente todas las variables, se observó un importante cambio en el patrón de influencia de las propias de la TCP. Así, para conocer las posibles causas, se procedió a la realización de un análisis de mediación y se propuso un modelo explicativo mediante path analysis con AMOS 20.

\section{Resultados}

En la Tabla 1 se presentan los descriptivos, los coeficientes $\alpha$ de fiabilidad y las correlaciones entre las variables. Los coeficientes $\alpha$ son todos de alta magnitud (entre $.77 \mathrm{y}$ .89), salvo los del CCP que es moderado (.65) y el de la NS (.54). También se observa que todas las variables analizadas presentan relaciones significativas con la variable dependiente. Cabe resaltar las correlaciones de la $\mathrm{CP}\left(\mathrm{r}^{2}=\right.$ .86) y la del CCP $\left(\mathrm{r}^{2}=-.77\right)$.

En lo que respecta a los datos de las regresiones jerárquicas en la predicción de la Intención (Tabla 2), en el Paso 1 se observa que las variables de la TCP son todas significativas y explican el $59 \%$ de la varianza $\left(\mathrm{F}_{(3,198)}=95.59 ; \mathrm{p}<\right.$ .0001). En el Paso 2, al introducir las nuevas variables, se produce un aumento en la varianza explicada con respecto al Paso $1\left(\mathrm{r}^{2}=.79\right)$, contribuyendo todas ellas de forma significativa (excepto la NS) al incremento de la varianza en un $20 \%\left(\mathrm{~F}_{(7.194)}=106.78 ; \mathrm{p}<.0001\right)$ y a la mejora de la capacidad predictiva del modelo original. Se observa, además, una modificación en el patrón de influencia de los constructos propuestos por Ajzen, ya que la actitud incrementa su rol explicativo cuando se analizan conjuntamente con las variables añadidas, sucediendo lo contrario con las tras dos variables, NS y CCP.

Tabla 1. Medias, desviaciones típicas, alphas y correlaciones

\begin{tabular}{|c|c|c|c|c|c|c|c|c|c|c|}
\hline & $\mathrm{M}$ & $\mathrm{SD}$ & $\alpha$ & Intención & Actitud & NS & $\mathrm{CCP}$ & NM & EPA & ENA \\
\hline Intención & 3.11 & 2.23 & .86 & & & & & & & \\
\hline Actitud & 5.91 & .94 & .87 & $-.38 * *$ & & & & & & \\
\hline NS & 5.87 & 1.05 & .54 & $-.65^{* *}$ & $.45^{* *}$ & & & & & \\
\hline $\mathrm{CCP}$ & 4.43 & 1.26 & .65 & $-.77 * *$ & $.59 * *$ & $.63^{* *}$ & & & & \\
\hline $\mathrm{NM}$ & 4.36 & 1.69 & .77 & $-.49 * *$ & $.31 * *$ & $.32 * *$ & $.41 * *$ & & & \\
\hline EPA & 1.17 & 1.02 & .80 & $.29 * *$ & $-.45 * *$ & $-.16^{*}$ & $-.30 * *$ & $-.15^{*}$ & & \\
\hline ENA & 3.19 & 1.72 & .89 & $-.38 * *$ & -.02 & $.31 * *$ & $.15^{*}$ & $.47 * *$ & -.03 & \\
\hline $\mathrm{CP}$ & 3.57 & & --- & $.86^{* *}$ & $-.46^{* *}$ & $-.63 * *$ & $-.81 * *$ & $-.42 * *$ & .11 & $-.28 * *$ \\
\hline
\end{tabular}


Tabla 2. Regresión jerárquica con las variables de la TCP, Emociones positivas y negativas, Norma Moral y Conducta Pasada

\begin{tabular}{|c|c|c|c|c|c|}
\hline & Paso 1 & Paso 2 & & & \\
\hline & $\beta$ & $\mathrm{R} 2$ & B & $\Delta \mathrm{R} 2$ & R2 \\
\hline Actitud & $.126^{*}$ & & $.166^{* * *}$ & & \\
\hline NS & $-.199 * *$ & & -.030 & & \\
\hline $\mathrm{CCP}$ & $-.70 * * *$ & $.59 * * *$ & $-.133 *$ & & \\
\hline EPA & & & $.220 * * *$ & $.014 * *$ & \\
\hline ENA & & & $-.099 *$ & $.041 * * *$ & \\
\hline NM & & & $-.097 *$ & $.014 * *$ & \\
\hline $\mathrm{CP}$ & & & $.703 * * *$ & $.134 * * *$ & $.79 * * *$ \\
\hline
\end{tabular}

Nota. $(\mathrm{NS}=$ Norma Subjetiva; $\mathrm{CCP}=$ Control Conductual Percibido; $\mathrm{NM}=$ Norma Moral; EPA

$=$ Emociones Positivas Anticipadas; ENA $=$ Emociones Negativas Anticipadas; $\mathrm{CP}=$ Conducta

Pasada) $* \mathrm{p}<.05 ; * * \mathrm{p}<.01 ; * * * \mathrm{p}<.001$

Teniendo en cuenta lo anterior y siguiendo las recomendaciones de Baron y Kenny (1986), parece que parte del cambio en la influencia del componente actitudinal sobre la intención podría estar mediado por la anticipación de reacciones afectivas positivas y la experiencia previa de los individuos en la realización de la conducta. De este modo, se llevó a cabo un análisis mediacional empleando como variable independiente la actitud hacia la conducta y como posibles variables mediadoras las emociones positivas anticipadas (EPA) y la conducta pasada (CP).

Se comprobó que el peso de regresión de la Actitud sobre la Intención $\left(\mathrm{B}_{1}=-.380\right)$ explica una varianza del $14 \%$. Al incluir las EPA, dicho peso bajó $\left(\mathrm{B}_{1}{ }^{\prime}=-.310\right)$, lo que podría indicar que estaba actuando como variable mediadora de la relación actitud hacia la conducta - intención. La prueba de Sobel (1982) corroboró lo anterior, mostrando un efecto significativo de esa mediación $\left(\mathrm{Z}_{(\text {(Sobel) }}=1.96 ; \mathrm{p}=.024\right)$. El cociente $\mathrm{B}_{1}-\mathrm{B}_{1}{ }^{\prime} / \mathrm{B}_{1}=.19$ informa que el $19 \%$ de la relación entre actitud e intención es explicada por la variable EPA.

Por su parte, la CP también media en esa relación pues se observó que el peso regresión baja $\left(\mathrm{B}_{1}{ }^{\prime}=.044\right) . \mathrm{Z}=$ $-2.54 ; \mathrm{p}=.005$. El cociente $\mathrm{B}_{1}-\mathrm{B}_{1}{ }^{\prime} / \mathrm{B}_{1}=.88$ indica que el $88 \%$ de la relación actitud-intención es explicado por la CP.

Para finalizar, se realizó un path analysis con todas las variables. Dado que las ENA no ejercieron un efecto significativo sobre la intención y que el modelo resultante no ofrecía un buen ajuste, se realizó uno final excluyéndola (Figura 1).

En la Figura 1 se puede observar que existe una influencia directa de las $\mathrm{EPA}(\beta=.23)$, la $\mathrm{NM}(\beta=-.14)$, la $\mathrm{CP}(\beta=.72)$ y la actitud $(\beta=.20)$ sobre la intención. Las EPA, al igual que la $\mathrm{CP}$, también ejercen una influencia indirecta sobre la intención a través de la actitud y el CCP. La varianza total explicada es del $79 \%$ y el ajuste del modelo es bueno $\left(\chi^{2}\right.$

$$
\begin{aligned}
& =5.26 ; \text { g.l. }=4 ; p=.262 ; \chi^{2} / \text { g.l. }=1.315 ; \mathrm{GFI}=.993 ; \mathrm{CFI} \\
& =.998 ; \operatorname{RMSEA}=.040[.000-.120]) .
\end{aligned}
$$

\section{Discusión}

Tradicionalmente se ha discutido si, como defiende Ajzen, la posible influencia de las emociones queda recogida en el concepto de actitud hacia la conducta. En nuestra opinión estos resultados parecen ir más allá de ese debate, pues no sólo se observa la necesidad de diferenciarlos claramente, sino que, además, se destaca que las emociones positivas tienen una influencia directa y significativa sobre la intención.

Un objetivo fundamental de este trabajo ha sido conocer la posible contribución que las emociones (positivas y negativas), la norma moral y la conducta pasada tienen en la explicación de la intención de fumar. Por un lado, se constata que las variables propuestas contribuyen significativamente al incremento de la varianza explicada del modelo original. Esto es, los sujetos que más fumaron durante los dos meses precedentes, que anticipan reacciones positivas de dicha conducta, que no sienten una presión moral para no realizarla y que tienen una puntuación más alta en la actitud hacia la conducta, esto es, una actitud negativa, son los que tienen una mayor intención de seguir fumando. Así, el fumar se percibe como malo y perjudicial, sin embargo no es motivo suficiente para plantearse el abandono del tabaco.

Cabe señalar aquí que, a diferencia de trabajos como los de Rivis, Sheeran y Armitage (2009), las emociones anticipadas positivas tienen una relación más fuerte con la intención que las negativas, lo que posiblemente sea debido a que lo que se experimenta al fumar es más importante que las repercusiones futuras en la salud.

La conducta pasada, al igual que en el trabajo de Higgins y Conner (2010), fue el predictor más fuerte de la intención, 


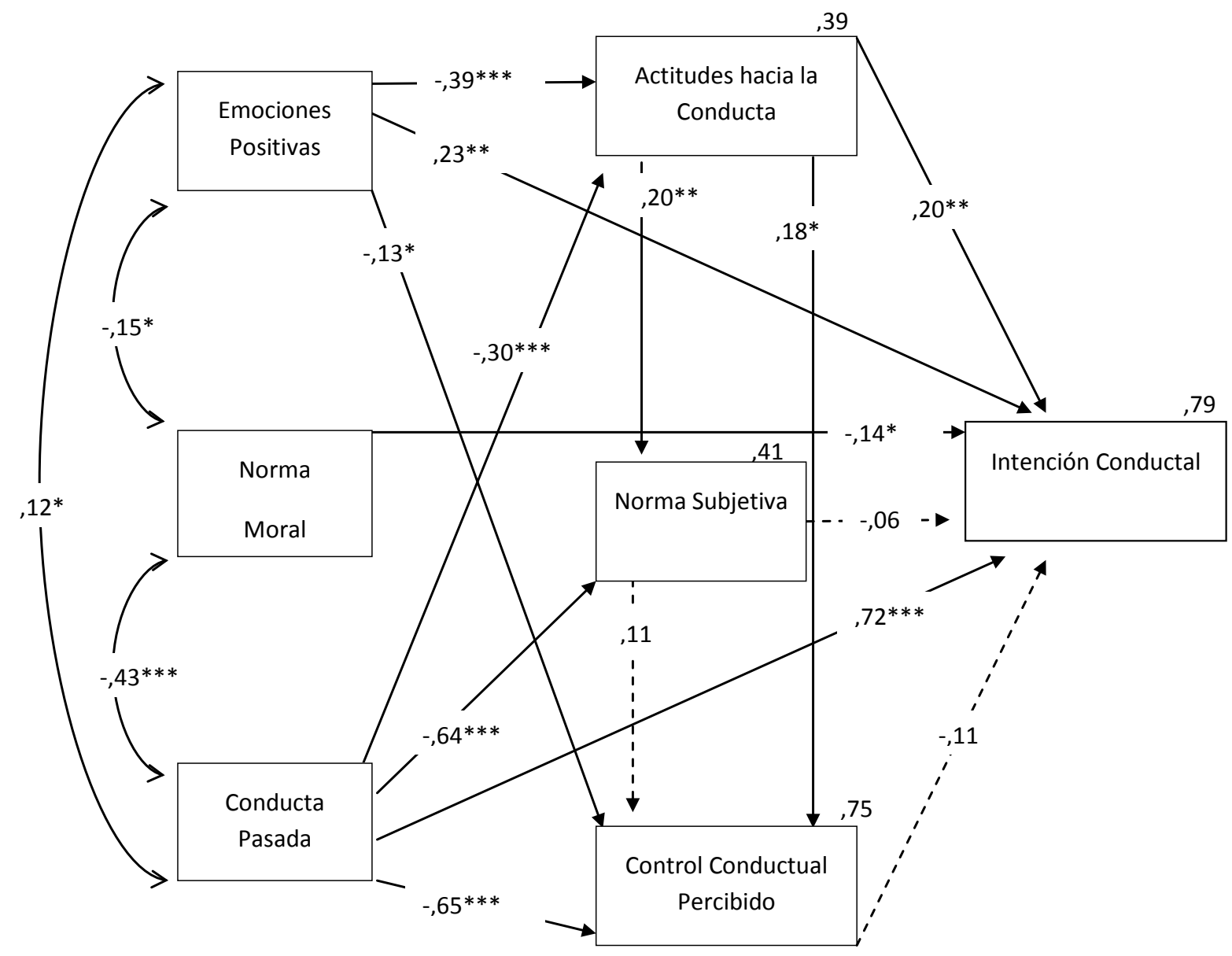

Figura 1. Modelo de Ecuación Estructural del modelo extendido de la TCP

lo que parece mostrar apoyo suficiente para proponerla como variable predictora de este comportamiento de riesgo, ya que se asoció directa y significativamente con la intención de seguir manteniendo dicha conducta.

Como se ha podido comprobar también, la inclusión de las nuevas variables provocó un cambio en las influencias de las variables originales de la TCP. Tanto la NS como el CCP disminuyeron su poder predictivo, mientras que la actitud hacia la conducta lo incrementó. Proponemos que dicho incremento es debido al papel mediador que desempeñan CP y las EPA en la relación actitud-intención.

Pero el modelo de ecuaciones estructurales propuesto, además de enfatizar lo anterior, también puso de manifiesto otro aspecto que consideramos muy destacable: las variables propuestas en este trabajo, no solo tienen una relación indirecta con la intención a través de los constructos de la TCP, como ya apuntaba Ajzen en muchos de sus trabajos, sino que también tienen una relación directa. Esto nos lleva a no poder reducir estas variables a simples relaciones residuales y poco significativas incluidas ya en la actitud y el Control Conductual Percibido. Esta relación directa abre un nuevo campo en el que investigar estas aportaciones más allá del modelo de la TCP. Se observa también que la NS y el CCP no tienen un papel significativo en la intención.

Podríamos por lo tanto asumir que la intención de fumar está más vinculada a los componentes emocionales positivos y de experiencia, que a cuestiones morales de presión social o de sentimientos de eficacia y controlabilidad de la acción. Lo que nos pone en el camino de los factores a tener en cuenta en la prevención del hábito de fumar y en la intervención para el cese de este hábito tan poco saludable. Incidir en las consecuencias negativas que el tabaco ocasiona en la salud a largo plazo es otro de los factores que habría que considerar en un programa de intervención.

Siendo conocedores de las características y limitaciones de la muestra y de que habría que probar todos estos resultados 
en muestras más amplias, una de las grandes limitaciones que hemos encontrado a la hora de realizar este trabajo ha sido la relacionada con el estudio de las emociones. Existe una gran heterogeneidad a la hora de concretar las evaluaciones afectivas entre los diferentes estudios (Conner, 2013). Coexiste una gran multiplicidad tanto en su selección, en las medidas utilizadas para evaluarlas como en la perspectiva de tiempo (unas de forma evocada y otras de forma anticipada), lo que dificulta enormemente la posible comparación que pueda llegar a establecerse con los resultados de otras investigaciones. Lo mismo sucede con la Conducta Pasada. En la comunidad científica hay una cierta discrepancia sobre cómo conceptualizar e interpretar la contribución de esta variable. Algunos investigadores ven el impacto de la conducta pasada fruto de un error de medición o como resultado de la presencia de otros factores que no se han tenido en cuenta (Ajzen 1991, 2002), mientras que otros ven la conducta pasada como una construcción significativa que es demasiado importante como para no tenerla en cuenta (Verplanken y Aarts, 1999). Nuestros resultados se suman a esta última perspectiva, considerando la conducta pasada como un elemento lo suficientemente relevante para incorporarlo en un modelo explicativo de intenciones y, muy posiblemente, también de futuras conductas.

Otra de las limitaciones es el hecho de que se ha focalizado el estudio en la intención conductual y no en la conducta en sí misma. Esto es, principalmente, porque, tal y como ya señalaba Ajzen y Sheikh (2013) en sus trabajos, las emociones, de estar relacionadas, lo estarían más con la intención de llevar a cabo una conducta que con la conducta en sí misma, por esta razon nos hemos decantado por el estudio de la intención. Futuras investigaciones deberían incluir también el estudio de la conducta propiamente dicha como variable importante a tener en cuenta y ver qué puede estar sucediendo en ese paso tan decisivo como es el de la relación intención-conducta.

\section{Referencias}

Ajzen, I. (1991). The Theory of Planned Behavior. Organizational Behavior and Human Decision Processes, 50, 179-211. doi: 10.1016/07495978(91)90020-T

Ajzen, I. (2002). Perceived behavioral control, self-efficacy, locus of control, and Theory of Planned Behavior. Journal of Applied Social Psychology, 32, 1-20.

Ajzen, I. (2006). Constructing a Theory of Planned Behavior Questionnaire. Recuperado desde http://people.umass.edu/aizen/tpb.html

Ajzen, I., y Madden, T. J. (1986). Prediction of goal-directed behavior: The role of intention, perceived control, and prior behavior. Journal of Experimental Social Psychology, 26, 305-328.
Ajzen, I., y Sheikh, S. (2013). Action versus inaction: anticipated affect in the theory of planned behavior. Journal of Applied Social Psychology, $43,155-162$.

Asociación Española Contra el Cáncer (2014). Información Tabaco. Recuperado desde https://www.aecc.es/sobreelcancer/prevencion/tabaco/ Paginas/Informaciontabaco.aspx

Becker, M. H. (1974). The health belief model and personal health behavior. Health Education Monographs, 2, 324-508.

Becoña, E. (2004). Monografía Tabaco. Introducción. Adicciones,. 16, 7-11.

Bermúdez, J., y Contreras, A. (2008). Predictores psicosociales del consumo de tabaco en adolescentes: extensión de la teoría de la conducta planificada. Revista de Psicopatología y Psicología Clínica, 13, 175-186.

Catalina, C., Gelpi, J. A., Cortés, M. V., y Martín, J. (2010). Evolución en España del consumo de tabaco en población trabajadora desde la entrada en vigor de la ley 28/2005 de medidas sanitarias frente al tabaquismo. Revista Española de Salud Pública, 84, 223-227.

Conner, M. (2013). Health cognitions, affect and health behaviors. The European Health Psychologist, 15, 33-39.

Conner, M., y Armitage, C. J. (1998). Extending the theory of planned behavior: A review and avenues for further research. Journal of Applied Social Psychology, 28, 1429-1464.

Conner, M., Sandberg, T., McMillan, B., y Higgins, A. (2006). Role of anticipated regret, intentions and intention stability in adolescent smoking initiation. British Journal and Health Psychology, 11, 85-101.

Dohnke, B., Weiss-Gerlach, E., y Spies, C. (2011). Social influences on the motivation to quit smoking: Main and moderating effects of social norms. Addictive Behaviors, 36, 286-293.

Fishbein, M., y Ajzen, I. (1975). Belief, Attitude, Intention, and Behavior: An Introduction to Theory and Research. Reading, MA: AddisonWesley.

Hassandra, M., Vlachopoulos, S., Kosmidou, E., Hatzigeorgiadis, A., Goudas, M., y Theodorakis, Y. (2011). Determinants of Intention to Smoke in a Sample of Greek Youth: The Role of School Grade Level and Parental Influences. Psychology and Health, 26, 1241-1258.

Higgins, A., y Conner, M. T. (2010). Long-term effects of implementation intentions on prevention of smoking uptake among adolescents: A cluster randomized controlled trial. Health Psychology, 29, 529-538.

Jellema, L., Abraham, C., Schaalma, H. P., Gebhardt, W., y Empelen, P. (2013). Predicting having condoms available among adolescents: The role of personal norm and enjoyment. British Journal of Health Psychology, 18, 453-468.

Lazuras, L., Chatzipolychroni, E., Rodafinos, A., y Eiser, R. (2012). Social cognitive predictors of smoking cessation intentions among smoker employees: the roles of anticipated regret and social norms. Addictive Behaviors, 37, 339-341.

McMillan, B., y Conner, M. (2003). Using the theory of planned behavior to understand alcohol and tabacco use students. Psychology, Health and Medicine, 8(3), 317-328.

McEachan, R., Conner, M., Taylor, N.J., y Lawton, R.J. (2011). Prospective prediction of health-related behaviors with the theory of planned behavior: A meta-analysis. Health Psychology Review, 5, 97-144. doi: 10.1080/17437199.2010.521684

Ministerio de Sanidad, Servicios Sociales e Igualdad de España (2011/12). Encuesta Nacional de Salud de España 2011/12. Recuperado desde https://www.msssi.gob.es/estadEstudios/estadisticas/encuestaNacional/ encuesta2011.htm

Moan, I., y Rise, J. (2005). Quitting smoking: Applying an extended version of the theory of planned behavior in predicting intention and behaviour. Journal of Applied Social Psychology, 10, 39-68.

Murnaghan, D.A., Blanchard, C.M., Rodgers, W.M., LaRosa, J.N., MacQuarrie, C.R., MacLellan, D.L. y Gray, B.J. (2010). Predictors of physical activity, healthy eating and being smoke-free in teens: A theory of planned behaviour approach. Psychology \& Health, 25, 925-941.

Natan, M., Viktoria, G., y Shamrai, V. (2010). Smoking during pregnancy: analysis of influencing factors using the Theory of Planned Behaviour. International Nursing Review, 57, 388-394. 
Rhodes, R. E., y Courneya, K. S. (2003). Modelling the theory of planned behavior and past behaviour. Psychology, Health y Medicine, 8, 57-69.

Rivis, A., Sheeran, P., y Armitage, C. J. (2009). Expanding the affective and normative components of the theory of planned behaviour: A metaanalysis of anticipated affect and moral norms. Journal of Applied Social Psychology, 39, 2985-3019. doi: 10.1111/j.1559-1816.2009.00558.x

Rosenstock, I. M. (1974). Historical origins of the health belief model. Health Education Monographs, 2, 1-8.

Spijkerman, R., van den Eijnden, R. J. J. M., Vitale, S., y Engels, R.C.M.E. (2004). Explaining adolescents' smoking and drinking behavior: The concept of smoker and drinker prototypes in relation to variables of the theory of planned behavior. Addictive Behaviors, 29, 1615-1622.

Verplanken, B., y Aarts, H. (1999). Habit, attitude, and planned behavior: Is habit an empty construct or an interesting case of goal-directed automaticity? In W. Stroebe \& M. Hewstone (Eds.). European review of social psychology (Vol. 10, pp. 101-134). Chichester, England: Wiley. 